\title{
Sui teoremi di convergenza delle medie nei processi non stazionari.
}

\author{
Nota I di Bruno Forte (a Pisa) (*)
}

Introduzione. - Dopo alcune premesse. intese a dare un certo ordine $\theta$ forma all'argomento, verranno anzitutto illustrati in questa prima nota i risultati, ritenati più importanti, che sinora sono stati conseguiti nella teoria ergodica dei processi stazionari. Saranno trattati in maniera più approfondita i teoremi di convergenza puntuale. Un esame critico degli enunciati di detti teoremi, condotto con l'ausilio di esempi, porrà in luce l'esigenza di ulte. riori ricerche su questo argomento. Di ciò ci si servirà anche quale naturale spunto per l'esposizione di risultati, che si ritengono nuovi, e che si concretizzano in due teoremi di convergenza più generali di quelli classici. Per meglio intendere l'utilità di quanto conseguito si è ritenuto opportuno esporre poi in una seconda nota alcune applicazioni significative dei metodi di indagine statistica dei fenomeni fisici, dove la teoria ergodica dei processi anzidetti trova un interessante e proficua applicazione.

§ 1. Difinizioni. - Siano $X$ un insieme di elementi $x$, F una o-algebra di sottoinsiemi di $X$, alla quale appartenga $X, m$ una misura definita in $\mathscr{F}$.

Si consideri una trasformaz!one $T$ da $X$ su $X$, misurabile, nel senso che l'immagine reciproca mediante la $T^{-1}\left({ }^{1}\right)$, di un qualunque insieme di $\mathfrak{F}$, appartiene ancora a $\mathfrak{F}$.

Si dirà processo elementare la quaterna $(X, F, m, T)$.

Due processi elementari $\left(X^{\prime}, \mathscr{F}^{\prime} m^{\prime} T^{\prime}\right)$ e $\left(X^{\prime \prime}, \mathscr{F}^{\prime \prime}, m^{\prime \prime}, T^{\prime \prime}\right)$ si diranno eguali, quando sia $X^{\prime}=X^{\prime \prime}, \mathscr{F}^{\prime}=\mathscr{F}^{\prime \prime}, m^{\prime}=m^{\prime \prime}, T^{\prime}=T^{\prime \prime}$ equivalenti nello spazio mensurale $\left(X^{\prime}, \mathscr{F}^{\prime}, m^{\prime}\right)\left({ }^{2}\right)$.

(*) Lavoro eseguito per la realizzazione del programma del gruppo dí ricerca n. 20 del C.N.R. (1960-61).

(1) Si ricordi che per immagine reciproca di un insiemo $A$, mediante una data trasfor. mazione $T$, si intende $l$ 'insieme formato da quegli elementi $x$, di $X$ la cui immagine $T x$ appartiene ad $A$.

(2) cfr. [1] pag. 6 . 
B. ForTe: Sui teoremi di convergenza delle medie nei processi, ece.

Si consideri ora una successione $\left(m_{k}\right)_{k \in K}$ di misure definite nella $\sigma$-algebra $\mathfrak{F}$ e una successione di trasformazioni misurabili $\left(T_{k}\right)_{k \in K}$ da $X$ su $X$, dove $K$ è l'insieme degli interi relativi

Si definisce processo discreio la successione dei processi elementari

$$
\left(\left(X, \mathfrak{A}, m_{k}, T_{k}\right)\right)_{k \in K} .
$$

È stazionario un processo quando sia ccstituito da processi elementari a due a due eguali. Un processo disereto si dirâ poi periodico di periodo $h$ (intero relativo) se è :

$$
\left(X, \mathfrak{F}, m_{k+h}, T_{k+h}\right)=\left(X, \mathfrak{F}, m_{k}, T_{k}\right) \text { per } k \in K
$$

E facile riconoscere che il prodotto di due o più trasformazioni misu. rabili è ancora una trasformazione misurabile. Ciò detto, si consideri, in relazione ad un dato provesso discreto, la trasformazione misorabile $T_{k}^{(i)}$ definita come segue;

$$
T_{k}^{(i)}=T_{k+i-1} \cdot T_{k+i-2} \ldots T_{k+1} \cdot T_{k}
$$

con $i \in N$ ( $N$ insieme degli interi positivi).

Per ogni $k \in K$ si chiamerà traiettoria di ordine $k$ dell' elemento $x \in X$, e la si indicherà con $\Gamma_{k}(x)$, la successione:

$$
x, T_{k} x, T_{k}^{(2)} x, \ldots, T_{k}^{(i)} x, \ldots
$$

In un generico processo disereto, per ogni elemento $x$ di $X$ si avrà quindi una infinità numerabile di traiettorie: una per ciascun valore dello intero $k$. In un processo stazionario risulta $\Gamma_{h}(x)=\Gamma_{k^{\prime}}(x)$ per $k \in K$ e $k^{\prime} \in K$ e quasi ovunque in $(X, \mathscr{F}, m)\left(m=m_{k}\right)$. In un processo periodico di periodo $h$ risulta $\Gamma_{k}(x)=\Gamma_{k+h}(x)$ per $k \in K$ quasi ovunque in $\left(X, \widetilde{g} ; m_{k}\right)$.

$\dot{\mathrm{E}}$ di facile verifica che la traiettoria $\Gamma_{l}(x)$ ha in comune con la traiet. toria $\Gamma_{k+, j}\left(T_{k}^{(j)} x\right)$ della immagine di $x$ mediante la $T_{k i}^{(j)}$ tutti gli elementi successivi al $j$-mo; basta invero osservare che è:

$$
T_{k+j}^{(i)}\left[T_{k}^{(j)} x\right]=T_{k}^{(i+j)} x
$$

per $k \in K, \quad i \in N, j \in N$.

Una funzione $p(x, k)$, a valori reali, definita in $X \times K$, si dirà un invariante nel processo discreto $\left(\left(X, \mathfrak{F}, m_{k}, T_{k}\right)\right)$ se per ogni $k \in K$ esiste un insieme $A_{k}$ di misura nulla nello spazio mensurale $\left(X, \mathfrak{F}, m_{k}\right)$, tale che:

$$
\varphi(x, k)=\varphi\left(T_{k}^{(i)} x, k+i\right) \quad \text { per } i \in N \text { e } x \in X-A_{k} .
$$


e per ogni $k \in K$ e ogni $i \in N$. Un invariante assume quindi lo stesso valore negli elementi $\left.\left(T_{k}^{(i)}(x), k+i\right)\right)$ di $X \times K$ semprechè $x$ appartenga ad $X-A_{k}$.

Un insieme $A d i$ F dicesi k-invariante nel proceşso $\left(X, \mathscr{F}, m_{k}, T_{k}\right)$ se, detta $X_{A}$ la sua funzione caratteristica, si ha:

$$
\chi_{A}(x)=\chi_{A}\left(T_{k}^{(i)} x\right) \quad \text { per } i \in N \text { ex } x \in X
$$

Un insieme $A$ di $\mathscr{F}$ si dirà poi invariante se è $k$-invariante per ogni $k \in K$.

Si dirà infine che in un processo $\left(\left(X, \mathfrak{F}, m_{k}, T_{k}\right)\right)$ si conserva la misura se per ogni insieme $A \in \mathscr{F}$ e per ogni $k \in K$ si ha:

$$
m_{k-1}(T-1)=m_{k}(A)
$$

\& 2 Invarianza del limite delle medie. I teoremi di convergenza puntuale. - Un primo problema, che si pone nello studio dei processi e che è pure di notevole importanza per le applicazioni, riguarda la ricerca di invarianti di particolare significato nei confronti del processo stesso ( $\left.{ }^{3}\right)$.

Un processo del tutto generale che porta alla costrazione di due invarianti a partire da una qualsivoglia funzione $f(x, h)$ definita in $X \times K$ è il seguente ( $\left.{ }^{4}\right)$.

Data la funzione $f(x, k)$, si consideri la media aritmetica

$$
\widehat{f}(x, k, n)=\frac{1}{n} \cdot \sum_{0}^{n-1} f\left(T_{k}^{(i)} x, k+i\right)
$$

dove, per comodità di scrittura, ̀̀ $T_{k}^{(o)} x=x$. Si ponga

$$
\begin{gathered}
\widehat{f}^{\prime}(x, k)=\lim _{n \rightarrow+\infty} \inf _{\hat{f}}(x, k, \mathrm{n}) \\
\widehat{f}^{\prime \prime}(x, k)=\lim _{n \rightarrow+\infty} \sup _{\hat{f}} \widehat{f}(x, k, n) .
\end{gathered}
$$

È agevole riconoscere che le funzioni $\widehat{f}^{\prime}(x, k)$ e $\widehat{f}^{\prime \prime}(x, k)$ cosi costruite sono dae invarianti nel processo discreto $\left(\left(X, \mathfrak{F}, m_{k}, T_{k}\right)\right)$ preso in esame.

(3) Si confronti questo problema con l'analogo classico relativo alla dinamiea analitiea [2] pag. 329 .

(4) Il procedimento esposto è anche l'unico preso finora in considerazione nella lettura sull' argomento. 
Ricordando la (1.4) si ha infatti :

$$
\begin{aligned}
\widehat{f^{\prime}}(x, k) & =\lim \inf \frac{1}{n}{ }^{n-1} \sum_{0}^{n-1} f\left(T_{k}^{(i)} x, k+i\right)= \\
& \left.=\lim \inf \frac{1}{n} \sum_{j}^{n-1} f T_{(k+j)}^{(i-j)}\left[T_{k}^{(i)}(x)\right], k+j+i-j\right)= \\
& =\lim \inf \frac{1}{m} \sum_{o}^{m-1} f\left(T_{k+j}^{(s)}\left[T_{k}^{(j)} x\right], k+j+s\right)=\bar{f}^{\prime}\left(T_{k}^{(j)} x, k+j\right)
\end{aligned}
$$

per ogni $j \in N$ ed ogni $x \in X$. La dimostrazione relativa a $\bar{f}^{\prime \prime}(x, k)$ è analoga. Di conseguenza anche il limite delle medie $(2 \cdot 1)$, quando per ogni $k$ esista quasi orunque in $\left(X, \mathscr{F}, m_{k}\right)$ e un invariante. Il particolare significato che nella trattazione statistica dei fenomeni fisici vione attribuito al limite delle medie $(2 \cdot 1)$ giustifica le recerche atte a stabilire condizioni sul processo $\left(X, \mathfrak{F}, m_{k}, T_{k}\right)$ e sulle funzioni $f(x, k)$ sufficienti per la convergenza quasi ovunque in $\left(X, \mathfrak{F}, m_{k}\right)$ delle loro medie $(2 \cdot 1)$.

Classicu a questo proposito è il teorema di D. G. BIRkHoff (6) "In ogni processo stazionario $(X, \mathfrak{F}, m, T)$ che conserva la misura, per ogni funzione $f(x)$ definita e sommabile in $X$ esiste quasi ovunque il limite $f(x)$ delle medie $\widehat{f}(x, n)$ ed è sommabile».

Pure acquisita è la dimostrazione della proposizione seguente $\left({ }^{\tau}\right)$ : "In ogni processo discreto $\left(X, \mathfrak{F}, m, T_{k}\right)\left({ }^{8}\right)$ nel quale si conservi la misura $\sigma$-finita, per ogni funzione numerica $f(x, k)$ definita in $X \times K$, sommabile in $X$ per ogni valore di $k$ e tale che la serie:

$$
\stackrel{+\infty}{\Sigma_{0}} \int_{X}|f(x, k+i)| d m
$$

sia convergente, esiste il limite $\widehat{f}(x, k)$ delle medie (2-1), per ogni $k \in K$, quasi ovunque in $X$. La funzione $\widehat{f}(x, k)$ è sommabile in $X$ per ogni $k \in K \gg\left({ }^{9}\right)$.

Si può facilmente riconoscere che questa ultima proposizione non

(5) Si vedano per questo [3] e [4] pag. 3 .

(6) Si veda [5] e [6], nonchè [1] pag. 18 e seg.

(7) Questo teorema, implicitamente contenuto in [8], è interpretato ed approfondito in [7].

(8) In un processo quindi $((X, F, m, T)$,$) nel quale la misura m_{k}$ non varia al variare di $k$ in $K$.

(9) Questi due teoremi sono noti con il nome di teoremi di convergenza puntuale. 
contiene il teorema di BrRKHoff; infatti seppure riguarda processi più generali dei processi stazionari, esclude, con la ipotesi di convergenza della serie $(2-4)$, la classe delle funzioni $f(x)$, definite e sommabili su $X$, che non dipendono da $k$. Le costanti, in particolare, per le quali certo esiste il limite delle medie $(2 \cdot 1)$ ovunque in $X$, non soddisfano l'ipotesi di convergenza della serie $(2 \cdot 4)$ e quindi non rientrano nella classe delle funzioni prese in considerazione dal teorema in questione.

Per tali motivi i risultati conseguiti con i teoremi sopra ricordati non possono essere ritenuti soddisfacenti: il primo perchè riguarda processi particolari quali quelli stazionari, il secondo, sia perchè riguarda processi non stazionari particolarissimi, sia perchè è limitato ad una classe di funzioni eccessivamente ristreth.

Si ritiene a questo punto utile illustrare con un esempio come in un processo $\left(\left(X, \mathfrak{F}, m_{k}, T_{k}\right)\right)$ di tipo genurale la sola ipotesi di sommabilità su $X$ delle funzioni $f(x, k)$, per $o_{i}$ ni $k \in K$, ipotesi certo soddisfatta dalle costanti, per misure $m_{k}$ finite, e dalle funzioni della sola $x$ sommabili, non sia sufficiente ad assicurare la esistenza del limite delle loro medie, quasi ovunque in $\left(X, F, m_{k}\right)$.

Si consideri il processo $\left(\left(X, \mathfrak{F}, m_{k}, T_{k}\right)\right)$; ove $X$ sia l'insieme dei reali dell' intervallo $(0,1), \mathscr{F}$ la famiglia degli insiemi di BoREL in detto intervallo, $m_{k}$ la ordinaria misura di LeBesgue DetTe $I$ e $I^{\prime}$ le trasformazioni, misurabili, di $(0,1)$ in sè, definile dalle relazioni:

$$
\begin{aligned}
& I x=x \text { per ogni } x \text { tale che } 0 \leq x \leq 1 \\
& I^{\prime} x=\begin{array}{l}
x+1 / 2 \text { per ogni } x \text { tale che } 0 \leq x<1 / 2 \\
x-1 / 2 \text { per ogni } x \text { tale ohe } 1 / 2 \leq x \leq 1
\end{array}
\end{aligned}
$$

e detto $H$ l'insieme delle potenze di 2 con esponente intero relativo, sia poi:

$$
\begin{array}{ll}
T_{k}=I^{\prime} & \text { per ogni } k \in H \\
T_{k}=I & \text { per ogni } k \in K-H
\end{array}
$$

Con ciò il processo $\left(\left(X, F, m_{k}, T_{k}\right)\right)$ è completamente caratterizzato.

Si consideri quindi la funzione sommabile $f(x)$ definita sull' intervallo $(0,1)$ come segue :

$$
f(x)=\quad\left\{\begin{aligned}
-1 & \text { per ogni } x \text { tale che } 0 \leq x<1 / 2 \\
1 & \text { per ogni } x \text { tale che } 1 / 2 \leq x \leq 1
\end{aligned}\right.
$$


Un calcolo immediato dà per essa:

$$
\widehat{f}\left(x, 0,2^{2 n+2}\right)=\frac{1}{3} \cdot \frac{2^{2 n+2}+2}{2^{2 n+2}}
$$

$$
f\left(x, 0,2^{2 n+1}\right)=-\frac{1}{3} \cdot \frac{2^{2 n+1}-2}{2^{2 n+1}}
$$

per ogni $x$ tale che $1 / 2 \leq x<1$,

$$
\begin{aligned}
& \widehat{f}\left(x, 0,2^{2 n+2}\right)=-\frac{1}{3} \cdot \frac{2^{2 n+2}+2}{2^{2 n+2}} \\
& \bar{f}\left(x, 0,2^{2 n+1}\right)=\frac{1}{3} \cdot \frac{2^{2 n+1}-2}{2^{2 n+1}}
\end{aligned}
$$

per ogni $x$ tale che $0 \leq x<1 / 2$

Di qui segue che per detta funzione nel processo considerato è ovunque sull' intervallo $[0,1]$ :

$$
\bar{f}^{\prime}(x, k) \leq-1 / 3, \quad 1 / 3 \leq \widehat{f}^{\prime \prime}(x, k)
$$

non esiste quindi il limite delle sue medie per ogni $x \in X$ ed ogni $k \in K$.

L'esempio ora esposto mostra perciò quanto segue: se si vuole che in un processo $\left.\left((X, F), m_{k}, T_{k}\right)\right)$ nel quale si conserva la misura esista il limite delle medie $(2-1)$ di ogni funzione $f(x, k)$ definita in $X \times K$, sommabile in $\left(X, \mathfrak{F}, m_{k}\right)$ per ogni valore di $k$, in particolare di ogni funzione della sola $x$ sommabile in $\left(X, \mathscr{F}, m_{k}\right)$, occorre formulare ulteriori ipotesi sul processo stesso.

Nel successivo paragrafo, rinunziando al ricorso diretto al teorema di BIRKHOFF, come è stato invece fatto in [7] e [8], bensì seguendo nelle linee generali il procedimento di Yosida E KaKuTant [9] e più da vicino quello di RIEsz [6], relativi ai processi stazionari, si otterranno due risultati, che se anche non esauriscono la questione, sono di per sè sufficientemente significativi.

§ 3. Teoremi ergodici massimali nei processi non stazionari. - Alla base delle considerazioni che verranno qui sviluppate è il seguente lemma elementare di RIESz $\left({ }^{10}\right)$ : "Data la successione finita di numeri reali :

$$
a_{1}, a_{2}, \ldots, a_{n}
$$

(10) Si veda [6] pag. 225. 
ed un intero positivo $m \leq n$, si considerino quei termini $a_{k}$ per ciascuno dei quali esiste almeno un intero potitivo $r \leq m,(r \leq n-k+1)$ tale che la successione finita:

$$
a_{k}, a_{k+1}, \ldots a_{k+,-1}
$$

soddisfa la relazione

$$
a_{k}+a_{k+1}+\ldots a_{k+2-1}>0
$$

La somma dei termini $a_{k}$ siffatti è positiva».

Dato il processo discreto $\left(\left(X, \mathscr{F}, m_{k}, T_{k}\right)\right)$ che conserva la misura, si consideri la funzione $f(x, k)$, a valori reali, definita in $X \times K$ e sommabile in $\left(X, \mathfrak{F}, m_{k}\right)$ per ogni $k \in K$. Si prenda poi in esame, per ogni coppia $(x, k) \in X \times K$ e per ogni coppia $(m, n)$ di interi positivi, la successione finita di numeri reali

$$
f(x, k), f\left(T_{k} x, k-1\right), \ldots, f\left(T_{k}^{(n+m-1)} x, k+n+m-1\right)
$$

Posto :

$$
\begin{gathered}
D_{m, k}^{(i)}=\left\{x: \text { esiste } r \leq m \text { tale che } f\left(T_{k}^{(i)} x, k\right)+\ldots+\right. \\
\left.+f\left(T_{k}^{(i+r-1)} x, k+i-1\right)>0\right\}
\end{gathered}
$$

Per il lemma di RIEsz si può affermare che detta $g_{m, k}^{(i)}$ la funzione caratteristica di $D_{m, k}^{(i)}$ risulta :

$$
\left.s_{m}(x, k)={\underset{0}{\Sigma_{i}}}_{\Sigma_{1}}^{n+m-1} f\left(T_{k}^{(i)} x, k+i\right) . g_{m, k}^{(i)}(x)\right)>0 \text { per }(x, k) \in X \times K .
$$

Conseguentemente la funzione $s_{m}(x, h)$ è sommabile in $\left(X, \mathscr{F}, m_{k}\right)$ per ogni $k \in K$ e si ha:

$$
\int_{X} s_{m}(x, k) d m_{k}>0 \quad \text { per } k \in K
$$

ovvero

$$
{\stackrel{n}{\Sigma_{i}}}_{\Sigma_{D_{m, k}^{(i)}}^{-1}} f\left(T_{k}^{(i)} x, k+i\right) d m_{k}+{\underset{n}{\Sigma_{j}}}_{D_{m+n-j, k}^{(j)}} f\left(T_{k}^{(j)} x, k+i\right) d m \geq 0
$$

Gli insiemi $D_{m, k}^{(i)}$, d'altra parte, soddisfano la seguente proprietà: se $x$ è un elemento di $D_{m, k}^{(i)}$ la sua immagine $T_{k} x$ appartiene a $D_{m, k+1}^{(i-1)}$ e vice- 
versa se $x \in D_{m, k+1)}^{(i-1)}$ eे anche $T_{k}^{-1} x \in D_{m, k}^{(1)} ;$ infatti: se $x \in D_{m, k}^{(i)}$, risulta (cfr. (3-2) :

$$
f\left(T_{k+1}^{(i-1)}\left[T_{k} x\right], k+1+i-1\right)+\ldots+f\left(T_{k-1}^{(i+r-2)}\left[T_{k}^{\prime} x\right], k+1+i+r-2\right)>0
$$

per almeno un $r \leq m$ e conseguentemente $T_{k} x \in D_{m, k-1}^{(i-1)}$; viceversa se $x \in D_{m, k+1}^{(i-1)}$ è per tale elemento

$$
f\left(T_{k+1}^{(i-1)} x, k+i\right)+\cdots+f\left(T_{k+1}^{(i+2)} x, k+i+r-1\right) \gtrless 0
$$

per almeno un $r \leq m$, o, ciò che ¿̀ lo stesso,

$$
f\left(T_{k}^{(i)}\left[T_{k}^{-1} x\right], k+i\right)+\ldots+f\left(T_{k}^{(i+r-1)}\left[T_{k}^{-1} x\right], k+i+r-1\right) \geq 0
$$

e conseguentemente $T_{k}^{-1} x \in D_{m, k}^{(i)}$. Si ha così che è $T_{k} D_{m, k}^{(i)}=D_{m, k+1}^{(i-1)}$ (11).

Poichè nel processo considerato $\left(\left(X, \mathscr{F}, m_{k}, T_{k}\right)\right)$ si conserva per ipotesi la misura, si avrà

$$
m_{k}\left(D_{m, k}^{(i)}\right)=m_{k+1}\left(D_{m, k+1}^{(i-1)}\right)
$$

$\mathrm{e}$ in generale:

$$
m_{k}\left(D_{m, k}^{(i)}\right)=m_{k+i}\left(D_{m, k+i}^{(0)}\right)
$$

Si indicbí con $E_{m, k+i}$ tale insieme $D_{m, k+i}^{(0)}$, cioè l'insieme

$\left\{x\right.$ : esiste $r \leq m$ tale che $f(x, k+i)+f\left(T_{k} x, k+i+1\right)+\ldots+$

$$
\left.+f\left(T_{k}^{(r-1)} x, k+i+r-1\right)>0\right\}
$$

In virtù di quanto sopra osservato si ha:

$$
D_{m, k}^{(i)}=\left[T_{k}^{(i)}\right]^{-1} E_{m, k_{i}} \text { e } m_{k}\left(D_{m, k}^{(i)}=m_{k+i}\left(E_{m, k+i}\right)\right.
$$

(11) Si ha anche $D_{m, k}^{(i)}=T_{k}^{-1} D_{m, k+1}^{(i-1)}$, si osservi a questo poposito che se è $A=T_{k} B$ in generale non d̀ anche $B=T_{k}^{-1} A$, non avendo formulato l'ipotesi di invertibilità ov. vero biunirocità nei riguardi delle trasformazioni $T_{k}$. 
B. Fonte: Sui teoremi di fonvergenzu delle medie nei processi, ecc. 271

applicando ora a ciascuno degli integrali che figurano nella relazione (3-5) il teorema di integrazione per sostituzione (12) si ha:

$$
{\stackrel{n-1}{\Sigma_{i}}}_{0}^{n-1} \int_{E_{m}, k+i} f(x, k+i) d m_{k+i}+\sum_{n}^{n+m-1} \int_{E_{m+n-j}, k+j} f(x, k+j) d m_{k+j} \geq 0
$$

e a maggior ragione:

$$
{\stackrel{n}{\Sigma_{i}}}_{0}^{n-1} \int_{E_{m}, k+i} f(x, k+i) d m_{k+i}+\sum_{n}^{n+m-1} \int_{X}\left|f(x, k+j) d m_{k+j}\right| \geq 0
$$

Poniamo $E_{k+i}=\lim _{m \rightarrow+\infty} E_{m, k+i}=\bigcup_{m \in N} E_{m, k+i}$. Tale insieme sarà percio costituito da quegli elementi $x \in X$ per i quali esiste almeno un intero positivo $m$ tale

$$
{\underset{0}{\Sigma}}_{i}^{m-1} f\left(T_{k}^{(i)} x, k+i\right)>0
$$

Usufruendo della (3-8) si conseguono i seguenti due lemmi $(A)$ e $(B)$, che seguendo la letteratura, possono essere catalogati tra i teoremi ergodici massimali.

Lemma $(A)$ : In un processo discreto periodico $\left(X, \mathfrak{F}, m_{k}, T_{k}\right)$ nel quale si conserva la misura, per ogni funzione $f(x, k)$ definita in $X \times K$ periodica in $k$ di periodo $h$ eguale a quello del processo, sommabile in $\left(X, \mathscr{F}, m_{k}\right)$ per ogni $k \in K$ si ha:

$$
\frac{1}{h}{\stackrel{h}{\Sigma_{i}}}_{0}^{h i} \int_{E_{k+i}} f(x, k+i) d m_{k+i} \geq 0
$$

Leima $(B)$ : Dato un processo discreto $\left(\left(X, \not{F}, m_{k}, T_{k}\right)\right)$ nel quale si conservi la misura, ed una funzione $f(x, k)$ definita su $X \times R$, sommabile in $\left(X, \mathscr{F}, m_{k}\right)$ per ogni $k \in K$ e tale che sia

$$
\lim _{n \rightarrow+\infty}{\stackrel{n+m}{\Sigma_{i}}}_{n}^{n+1}|f(x, k+i)| d m_{k+i}=0
$$

uniformemente rispetto ad $m$, si ha:

$$
\lim _{n \rightarrow+\infty} \inf _{n} \frac{1}{n} \sum_{0}^{n-1} \int_{E_{k+i}} f(x, k+i) d m_{k+i} \geq 0
$$

(12) Si veda [10] pag. 163. 
Dimostrazione del lemma $(A)$ : Per le ipotesi di periodioità formulate nei riguardi del processo e della funzione presa in considerazione si ha: $f(x, k+s . h)=f(x, k)$ per ogni $(x, k)$ in $X \times K$ e per ogni $s \in N, T_{k+s . h}$ e $T_{k}$ equivalenti per ogni $s \in N$ nello spazio mensurale $\left(X, \mathfrak{F}, m_{k}\right)$. Relazione d' equivalenza quest' ultima la quale implica anche l'equivalenza in $\left(X, \mathscr{F}, m_{k}\right)$ delle funzioni caratteristiche

$$
\chi_{E_{m}, k+s \cdot h}, \chi_{E_{m}, k}
$$

rispettivamente di $E_{m, k+s h}$ e $E_{m}, k$.

Dalla (3.8) per $n=s . h$, si ha:

$$
\begin{aligned}
& \frac{s}{s \cdot h} \stackrel{h-1}{\Sigma_{0}} \int_{E_{m},} f(x, k+i) d m_{k+i}+ \\
& +\frac{1}{s . h} \underset{s h}{\sum_{i} h+f_{i}} \int_{X}^{n h-1}|f(x, k+j)| d m_{k+i} \geq 0
\end{aligned}
$$

Poichè :

$$
\frac{1}{s . h} \underset{\substack{s . h \\ \Sigma_{j}}}{s+h} \int_{X}|f(x, k+j)| d m_{k+j}
$$

$\grave{e}$ indipendente da $s$, risulta

$$
\frac{1}{h}{\stackrel{h}{\sum_{i}}}_{0-1}^{h-1} \int_{E_{m}, k+i} f(x, k+i) d m_{k+i} \geq 0
$$

cio che implica:

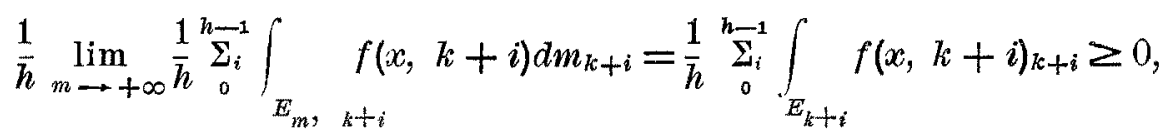

cioè l'asserto.

Dimostrazione deL lemma $(B)$ : Dalla ipotesi (3-10) segue che per ogni $\varepsilon>0$ si può determinare un intero, positivo, $\bar{n}(\varepsilon)$ tale che per ogni 
$n \geq \bar{n}$ e qualunque sia $m$ si abbia:

$$
\frac{1}{n} \stackrel{n-1}{\Sigma_{i}} \int_{E_{m}, k+i} f(x, k+i) d m_{k+i} \geq-\varepsilon
$$

sarà quindi anche:

$$
\begin{aligned}
& \left.\lim _{m \rightarrow+\infty} \frac{1}{n} \underset{0}{\sum_{i}^{n-1}}\right|_{E_{m}, k+i} f(x, k+i) d m_{k+i}= \\
& =\frac{1}{n}{\stackrel{n}{n} \sum_{i}^{-1}}_{E_{k+i}} f(x, k+i) d m_{k+i} \geq-\varepsilon
\end{aligned}
$$

ed ancora :

$$
\lim _{n \rightarrow+\infty} \inf \frac{1}{n} \sum_{0}^{n-1} \int_{E_{k+i}} f(x, k+i) d m_{k+i} \geq-\varepsilon
$$

e, per l' arbitrarietà di $\varepsilon$,

$$
\left.\lim _{n \rightarrow+\infty} \inf \frac{1}{n}{\stackrel{n}{\Sigma_{i}}}_{0}^{n}\right|_{E_{k+i}} f(x, k+i) d m_{k+i} \geq 0
$$

ciò l' asserto.

\$ 4. Teoremi di convergenza puntuale. - Dato un processo $\left.\left((X, F) m_{k}, T_{k}\right)\right)$ in cui si conservi la misura $\theta$ una funzione $f(x, k)$ definita in $X \times K$ e misurabile. Prendiamo in esame i seguenti sottoinsiemi, misurabili, di $X$. Fissati due numeri razionali $\alpha$ e $\beta$ con $\alpha<\beta$, consideriamo l'insieme:

$$
\begin{aligned}
Y_{k, \alpha, \beta} & =\left\{x: \lim _{n \rightarrow+\infty} \inf _{n} \frac{1}{n} \sum_{0}^{n-1} f\left(T_{(k)}^{(i)} x, k+i\right)<\alpha<\right. \\
& \left.\left.<\beta<\lim _{n \rightarrow+\infty} \sup _{n} \frac{1}{n}{\stackrel{\Sigma}{\Sigma_{i}} f}_{0}^{n-1} T_{k}^{(\ell} x, k+i\right)\right\}
\end{aligned}
$$

Per la invarianza del minimo e massimo limite delle medie che ivi figurano segue

$$
Y_{k, a, \beta}=T_{k}^{-1} Y_{k+1, a, \beta}
$$


e quindi

$$
m_{k}\left(Y_{k, \alpha, \beta}\right)=m_{k+1}\left(Y_{k+1}, \alpha, \beta\right)
$$

Si riconosce inoltre ohe per ogni fissato $k$ linsieme $Y_{k}$ unione numerabile di tutti gli insiemi $Y_{k, \alpha, \beta}$ che si hanno al variare di $\alpha$ e $\beta$ nel campo dei numeri razionali è formato da tutti e soli gli elementi $x$ in $X$ per i quali è:

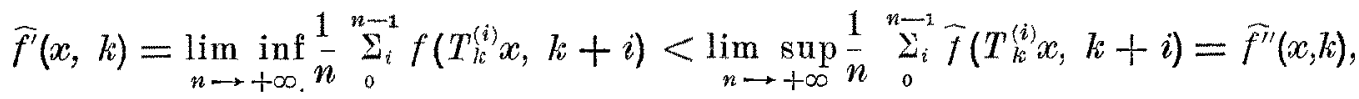

e cioè formato da tutti e soli quegli elemənti $x$ in $X$ per i quali non esiste il limite delle medie (2.1) della data funzion $f(x, k)$.

Si osservi in particolare che come per gli insiemi $Y_{k, a, \beta}$ anche per gli insiemi $Y_{k}$ si ha:

$$
Y_{k}=T_{k}^{-1} Y_{k+1}
$$

$\theta$ quindi anche:

$$
m_{k}\left(Y_{k}\right)=m_{k+1}\left(Y_{k+1}\right)
$$

Con queste premesse ed in virtù dei lemmi $(A)$ e $(B)$, rispettivamente, si provano i seguenti teoremi di convergenza puntuale:

Teorema $(A)$ : In un processo periodico di periodo, intero, $h$ con misure $m_{k} \sigma$-finite che si conservano, per ogni funzione $f(x, k)$ definita $X \times K$, periodica in $k$, di periodo $h$. sommabile su $X$, per tutti i $k \in K$, si ha:

$$
\widehat{f}^{\prime}(x, k)=\widehat{f^{\prime \prime}}(x, k)
$$

per ogni $k \in K$ e a meno di un insieme $Y_{k}$ di elementi $x$, in $X$, con $m_{k}\left(Y_{k}\right)=0$.

Trorema (B): Dato un processo $\left(\left(X, F, m_{k}, T_{k}\right)\right)$ nel quale le misure si conservino e siano o-finite, e data una funzione $f(x, k)$ definita su $X \times K$, sommabile su $X$, per tutti i valori di $k \in K$ e tale ehe sia

$$
\lim _{n \rightarrow+\infty} \frac{1}{n} \sum_{n}^{n+m-1} \int_{X}|f(x, k+j)| d n_{k+j}=0
$$

uniformemente rispetto all' intero $m$, si ha:

$$
\widehat{f}^{\prime}(x, k)=f^{\prime \prime}(x, k)
$$

a meno di un insieme $Y_{k}$, con $m_{k}\left(Y_{k}\right)=0$, e per ogni $k \in K$. 
Dimostrazione Del teorema $(A)$ : Senza ledere la generalità del risul. tato ci si può limitare al caso in cui è $f(x, k) \leq 0$ per ogni $(x, k)$ in $X \times K$. Sia quindi $f(x, k)$ una funzione siffatta che soddisfi inoltre le ipotesi precisate per essa nell' enunciato del teorema (A). Basterà perciò provare che per ogni fissata coppia di numeri razionali non negativi $\alpha$ e $\beta$ con $\alpha<\beta$ e per ogni $k \in K$, è :

$$
m_{k}\left(Y_{k, \alpha, \beta}\right)=0
$$

Si cominci col provare che tale insieme $Y_{k, x, \beta}$ ha misura finita. Poichè la misura $m_{k}$ presa in considerazione è $\sigma$-finita è sufficiente per questo riconoscere che l'insieme delle misure di tutti gli insiemi misurabili e di

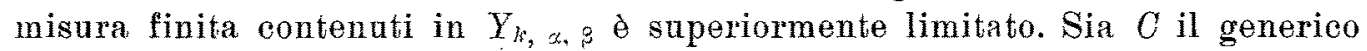
di tali insiemi, sia $\chi_{e}(x)$ la sua funzione caratteristica, per ogni $x \in Y_{k, \alpha, \beta} \mathrm{e}$ quindi per ogni $x \in C$ è $\bar{f}^{\prime \prime}(x, k)>\beta$; si può quindi trovare un intiero $m$ per il quale sia

$$
\frac{1}{m} \sum_{0}^{m-1} f\left(T_{k}^{(i)} x, k+i\right)>\beta
$$

ovvero

$$
\underset{0}{\Sigma_{i}} f\left(T_{k}^{(i)} x, k+i\right)-m \beta>0
$$

Tenuto conto che è $\beta>0$, per ogni $x$ in $C$ si avrà perciò, a maggior ragione

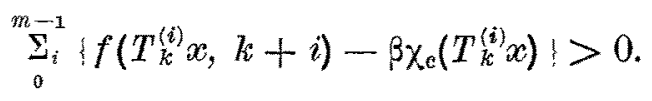

Applichiamo ora alla funzione:

$$
\psi(x, k)=f(x, k)-\beta \chi_{e}(x)
$$

definita e sommabile in $X$, il lemma $(A)$. È certo, in virtù della $(4-9) E_{k} \geq C$, si avrà così :

$$
\bar{h}{\stackrel{1}{\Sigma_{i}}}_{\Sigma_{i}}^{\Sigma_{i}} \int_{E_{k+i}}\left\{f(x, k+i)-\beta \chi_{c}(x)\right\} d m_{k+i} \geq 0
$$


e quindi

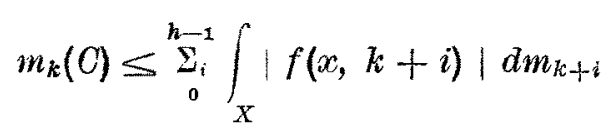

che prova quanto si voleva.

Si consideri ora la funzione caratteristica $\chi_{Y_{\alpha, \beta}}$ dell'insieme $Y_{\alpha, \beta}=$ $=\bigcup_{k \in K} Y_{k, \alpha, \beta}$ e si sostituiscano alle misure $m_{k}$ nel dato processo periodico $\left(\left(X, \mathscr{F}, m_{k}, T_{k}\right)\right)$ le misure $\bar{m}_{k}$ definite per ogni insieme $A \in \mathscr{F}$ come segue:

$$
\overline{\boldsymbol{m}}_{k}(A)=\boldsymbol{m}_{k}\left(A \cap Y_{k, \alpha, \beta}\right)
$$

Si può agevolmente riconoscere che il nuovo processo $\left(\left(\bar{X}, \mathscr{F}, \bar{m}_{k}, T_{k}\right)\right.$ è ancora periodico, di periodo $h$, e che in esso si conserva la misura.

Per provare il teorema $(A)$ non resta che fare ulteriormente ricorso al lemmá corrispondente, applicando al processo $\left(\left(X, \mathscr{F}, \bar{m}_{k}, T_{k}\right)\right)$ e alla seguente funzione:

$$
\begin{array}{ll}
\theta_{1}(x, r)=f(x, r)-\beta \chi_{Y_{\alpha, \beta}} \underset{\left.x^{\prime}\right)}{ } & \text { per ogni } x \in Y_{\alpha, \beta} \\
\Theta_{1}(x, r)=0 & \text { per ogni } x \in X-Y_{\alpha, \beta}
\end{array}
$$

funzione periodica in $r$ di periodo $h, \bar{m}_{k}$-sommabile su $X$ per ogni $k \in K$, il lemma $(A)$; si ha:

$$
\frac{1}{h}{\stackrel{h}{\Sigma_{i}}}_{\Sigma_{E_{k+i}}}^{\sum_{k+i}} \Theta_{1}(x, k+i) d \bar{m}_{k+i} \geq 0
$$

D'altra parte e certo

$$
E_{k+i} \supseteq Y_{k+i, \alpha, \beta}
$$

e quindi per le (4-10)

$$
\bar{m}_{k+i}\left(E_{k+i}\right)=m_{k+i}\left(Y_{k+i, \alpha, \beta}\right)
$$

si avrà̀ così :

$$
\frac{1}{h}{\stackrel{h}{\Sigma_{i}} \int_{\gamma_{k+i}, \alpha, \beta}}\left\{f(x, k+i)-\beta\left\{d m_{k+i} \geq 0\right.\right.
$$


In maniera del tutto analoga, considerando la funzione:

$$
\begin{array}{ll}
\left.\Theta_{2}(x, r)=\alpha \gamma_{, Y \alpha, \beta}(x)-f x, r\right) & \text { per ogni } x \in Y_{\alpha, \beta} \\
\Theta_{2}(x, r)=0 & \text { per ogni } x \in X-Y_{\alpha, \beta} ;
\end{array}
$$

applicato ad essa il lemma $(A)$ si avrà:

$$
\frac{1}{h} \sum_{0}^{h-1} \int_{Y_{k+i}, \alpha, \beta}\{\alpha-f(x, k+i)\} d m_{k+i} \geq 0
$$

sommando questa disuguaglianza con la (3-23) si ha:

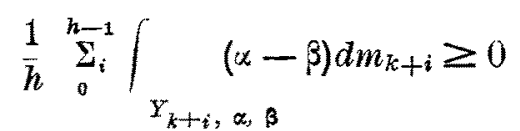

e quindi

$$
\frac{1}{h}{\underset{\Sigma}{\Sigma_{i}}(\alpha-\beta) m_{k+i}}_{\sum_{k+i, \alpha, \beta}}\left(Y_{k+1}=(\alpha-\beta) m_{k},\left(Y_{k, \alpha, \beta}\right) \geq 0\right.
$$

e poichè è $\beta>\alpha$ di qui si ha $m_{k}\left(Y_{k, \alpha, \beta}\right)=0$, il che prova l'asserto.

Dimostrazione del teorema $(B)$ : Sia $C$ un insieme di misura finita, del resto generico, contenuto in $Y_{k, \alpha, \beta}$, sia poi $\varphi_{c}(x, r)$ la funzione così definita in $X \times K$

$$
\begin{array}{ll}
\varphi_{c}(x, k)=1 & \text { per ogni } x \in C \\
\varphi_{c}(x, k)=0 & \text { per ogni } x \in X-C \\
\varphi_{c}(x, r)=0 & \text { per ogni } r \neq k \text { e } x \in X .
\end{array}
$$

Tale funzione è sommabile su $X$ per ogni $r \in K$, soddisfa inoltre l'ipotesi (4-6). Si applichi alla funzione

$$
\psi(x, r)=f(x, r)-\varphi_{\mathrm{c}}(x, r)
$$

che pure soddisfa la ipotesi anzidetta, il lemma $(B)$. Si riconosce operando per il resto in analogia con quanto fatto a proposito del teorema $(A)$ che la misura $m_{k}(C)$ è maggiorata da una quantità finita indipendente dall'insieme $C$ preso in considerazione. Ciò assicura che la misura $m_{k}\left(Y_{k, \alpha, \beta}\right)$ dell' insieme $Y_{k, \alpha, \beta}$ è finita. 
Si sostituisca ora il processo $\left(\left(X, \mathscr{F}, m_{k}, T_{k}\right)\right)$ con il processo $\left(\left(X, \mathfrak{F}, \bar{m}_{k}\right.\right.$, $\left.T_{k}\right)$ definito mediante le relazioni (4-10). Si applichi ancora il lemma $(B)$ alle funzioni così definite;

è

$$
\Theta_{1}(x, r)=f(x, r)-\beta g_{1}(x, r)
$$

$$
\Theta_{2}(x, r)=\alpha g_{2}(x, r)-f(x, r)
$$

con:

$$
\begin{array}{ll}
g_{1}(x, r)=1 \text { per ogni } x \in Y_{r}, \alpha, \beta & \text { con } r<\bar{n}(\varepsilon)+k \\
g_{1}(x, r)=0 \text { per ogni } x \in X-Y_{r, \alpha, \beta} & \text { con } r<\bar{n}(\varepsilon)+k \\
g_{1}(x, r)=0 \text { per ogni } r \geq \bar{n}(\varepsilon)+k \text { e per } x \in X & \text { con } r<\bar{n}(\varepsilon)+k \\
g_{2}(x, r)=1 \text { per ogni } x \in Y_{r, \alpha, \beta} & \text { con } r<\bar{n}(\varepsilon)+k \\
g_{2}(x, r)=0 \text { per ogni } x \in X-Y_{r, \alpha, \beta} & \text { con } r \geq \bar{n}(\varepsilon)+k \\
g_{2}(x, r)=\frac{2}{\alpha} f(x, r) \text { per ogni } x \in X &
\end{array}
$$

ove $\bar{n}(\varepsilon)$ è tale che per ogni $n \geq \bar{n}(\varepsilon)$ sia :

$$
\frac{1}{n} \sum_{n}^{n+m-1} \int_{X}^{n}|f(x, k+i)| d \mathrm{~m}_{k+i} \leq \varepsilon \text { per ogni } m \in N
$$

Si ha cosi, con procedimento analogo a quelle seguito a proposito del teorema $(A)$ :

$$
\frac{1}{n} \sum_{0}^{n-1} \int_{Y_{k+i}, \alpha, \beta}\{f(x, k+i)-\beta\} d m_{k+i} \geq-\varepsilon
$$

e, rispettivamente :

$$
\frac{1}{n} \sum_{0}^{n-1} \int_{Y_{k}+i, \alpha, \beta}\left(\alpha-f(x, k+i) ; d m_{k+i} \geq-\varepsilon\right.
$$

Dalle (4.13) e (4-14) si ha, per somma membro a membro:

$$
\frac{1}{n} \sum_{0}^{n-1}(\alpha-\beta) m_{k+i}\left(Y_{k+i, \alpha, \beta}\right)=(\alpha-\beta) m_{k}\left(Y_{k, \alpha, \beta}\right) \geq-2 \varepsilon
$$


l'arbitrarietà di $\varepsilon$ e l' essere $\beta>\alpha$ assicurano che è anche

$$
m_{k}\left(Y_{k, \alpha, \beta}\right)=0
$$

Si osservi che mentre il teorema $(A)$ può essere riguardato come una estensione del classico teorema di BiRKnoff, il teorema $(B)$ ora provato è una estensione del teorema esposto in [7]; infatti mentre la convergenza della (2-3) implica il verificarsi della (4.6), nei particolari processi nei quali la misura $m_{k}$ non varia al variare di $k$ il verificarsi della (4-6) non implica quello della (2.3).

Le due proposizioni, teorema $(A)$ e teorema $(B)$, sono indipendenti; è augurabile che si possa stabilire un teorema di convergenza puntuale che le contenga extrambe.

Si ritiene tuttavia che la esposizione dei tetti teoremi sia utile per un maggior approfondimento del problema.

\section{BIBIIOGRAFIA}

[1] P. R. Hacmos, Lectures on ergodic Theory, \& Math. Soc. of. Japan , 1956.

[2] T. Levi Crvita e U. Amald, Lezioni di Meccanica razionale, Zanichelli, Bologna, 1927

[3] J. Kampe de Feriet, La notion de moyenne dans la thérie de la turbolence, "Rend. Sem. Mat. e fisico di Milauo * vol. XXVII, 1955-56).

[4] A. Blanc -Lapierre, P. Casal A. Tortrat, Méthodes matematiques de la Mécanique, Masson \& Cie, éd. Paris (1959).

[5] D. G. Bıknoff, Proof of the ergodic theorem, Nat Acad Proc.», vol. 17, pp. 650-655, (dic. 1981).

[6] F.Rinsz, Sur la théorie ergodique, "Comm. Path. Helv. vol. 17, 221-229, (L944-45).

[7] B. Forre, Sulla convergenza delle medie temporali nella teoria ergodica dei fenomeni non stazionati; , Rivista di Matematica; Universita di Parma», (2), n. 1, 1960, pp. 29-44.

[8] S. KaxUTAN, Randon ergodie theorems and Markhoff processes with a stable distribution, Proc. of the second Berk. Symp., (195i), pag. 237 .

[9] Yosma e S. KaKUTaNI, Burkhf' s ergodic theorem and the maximal ergodic theorem, * Proc. Imp. Acad. Tokyo, 1939, 15, pp. 165-68.

[10] P. R. Hacmos, Measure Theory, ed. Van Nostrand Co. New York (1951). 